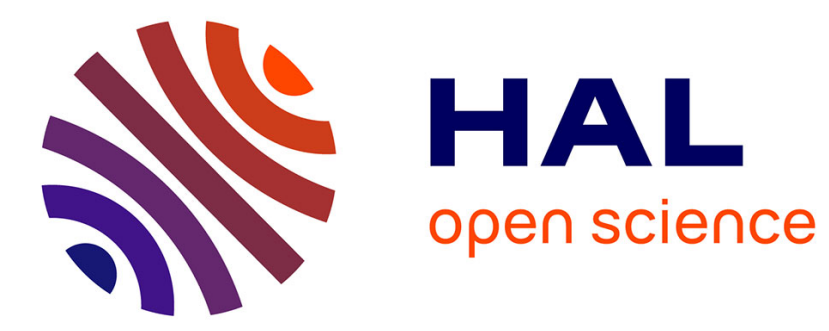

\title{
Composites structural modelling with uncertain data
}

\author{
N. Carrère, Y. Rollet, V. Retel, L. Boubakar, J.-F. Maire
}

\section{To cite this version:}

N. Carrère, Y. Rollet, V. Retel, L. Boubakar, J.-F. Maire. Composites structural modelling with uncertain data. Composites Science and Technology, 2009, 69 (1), pp.60. 10.1016/j.compscitech.2007.10.052 . hal-00575238

\section{HAL Id: hal-00575238 \\ https://hal.science/hal-00575238}

Submitted on 10 Mar 2011

HAL is a multi-disciplinary open access archive for the deposit and dissemination of scientific research documents, whether they are published or not. The documents may come from teaching and research institutions in France or abroad, or from public or private research centers.
L'archive ouverte pluridisciplinaire HAL, est destinée au dépôt et à la diffusion de documents scientifiques de niveau recherche, publiés ou non, émanant des établissements d'enseignement et de recherche français ou étrangers, des laboratoires publics ou privés. 


\section{Accepted Manuscript}

Composites structural modelling with uncertain data

N. Carrère, Y. Rollet, V. Retel, L. Boubakar, J.-F. Maire

PII:

S0266-3538(07)00441-1

DOI:

10.1016/j.compscitech.2007.10.052

Reference:

CSTE 3890

To appear in:

Composites Science and Technology

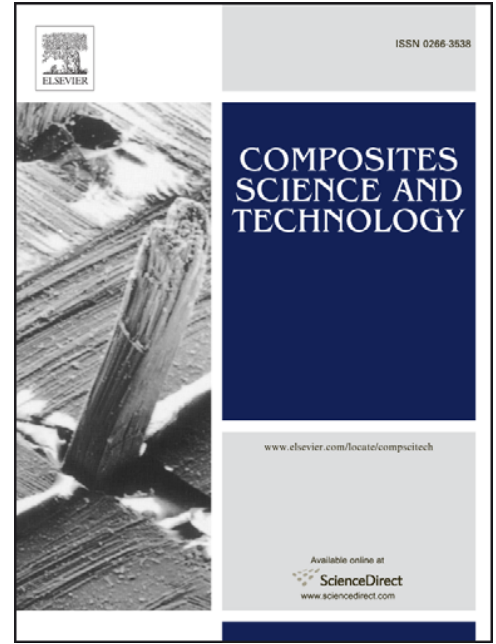

Received Date:

30 March 2006

Accepted Date:

11 October 2007

Please cite this article as: Carrère, N., Rollet, Y., Retel, V., Boubakar, L., Maire, J.-F., Composites structural modelling with uncertain data, Composites Science and Technology (2007), doi: 10.1016/j.compscitech. 2007.10.052

This is a PDF file of an unedited manuscript that has been accepted for publication. As a service to our customers we are providing this early version of the manuscript. The manuscript will undergo copyediting, typesetting, and review of the resulting proof before it is published in its final form. Please note that during the production process errors may be discovered which could affect the content, and all legal disclaimers that apply to the journal pertain. 


\section{Composites structural modelling with uncertain data}

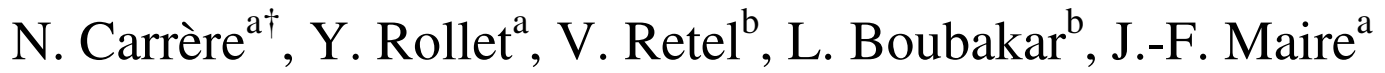

${ }^{a}$ ONERA (French Aerospace Research Agency), 29 avenue de la Division Leclerc, BP 72, F-92322 CHATILLON CEDEX

${ }^{\mathrm{b}}$ LMARC, 24 chemin de l'Epitaphe, F-25000 BESANCON

ABSTRACT :

The aim of this paper is to propose a methodology in order to take into account the influence of uncertain data in structural calculations. A specific method, based on the approximation of the responses as a function of the uncertain data, is proposed. Classical methods from the literature are also considered. The different methods are compared on a simple example in terms of response (average value and standard deviation) and in terms of computational cost. The method proposed in the present work permits to estimate correctly the whole response and is very simple to use (pre and post processing). This method is applied to a structural calculation on a "hatshapes" part called, manufactured in the quasi-isotropic IM7/977-2 composite.

Keywords : Structural calculations, Uncertain data, Variability

\section{Introduction}

The use of Polymer Matrix Composites (PMC) seems to be an essential evolution in terms of mass saving and innovative solutions. However, the available simulation tools, used during design of new structures, do not take into account their specific aspects (local heterogeneities, complexity of damage/rupture mechanisms, influence of the manufacturing process ...) that leads

\footnotetext{
${ }^{\dagger}$ Corresponding author : N. Carrere (carrere@onera.fr) tel. +3314673 46 47, fax. +331 46734691
} 


\section{ACCEPTED MANUSCRIPT}

to an under-using of these materials. A number of sources of lack in confidence could be evidenced (i) numerical aspects (mesh refinement, algorithms ...), (ii) numerical models (choice of the material behaviour, of the rupture criterion ...) and (iii) material uncertainties (materials parameters, boundary conditions). In order to obtain a sufficient degree of confidence, generous safety coefficients or important experimental campaigns are necessary, thus leading to maintain classical metallic solutions. The first two sources of uncertainties can be quantified and reduced, but the last one is partially "irreducible". So, it is of absolute necessity to understand the role and the influence of uncertainties on the structure behaviour. The aim of the present work is thus to propose a methodology that permits to perform structural modelling taking into account material uncertainties in order to predict their effects on the behaviour of the structure.

Data used in structural modellings are known only within a range of confidence (due for instance to the imperfections of the geometry or uncertainties on the loading conditions during structure tests, the natural variability of the mechanical properties ...). A number of methods exist in the literature to estimate the effects of uncertainties on the response of a structure :

- Calculation with the extreme data (called B-values) which requires only one evaluation of the response but provides only one pessimistic value without any statistical information on the obtained result.

- Sampling procedures (Monte-Carlo). The advantage of these methods is their simplicity of implementation, giving a good estimate of the interval of the responses but at a very high cost as soon as the number of variables increases.

- Stochastic Finite Element Modelling (SFEM) based on perturbation, spectral Neumann expansion, projection on a polynomial chaos are the most popular approaches widely 
developed during these last few years [1-3]. These methods require, in order to be applied practically, significant modifications of the computer code. Moreover, they are in general used only for variations of material properties.

The previous methods are the most popular to deal with problems of variability. It should also be noted that some authors develop approaches on non-determinist arithmetic operations (fuzzy [4], interval [5]).

In the present work, we propose a specific method which permits to study the effects of various kinds of material uncertainties (geometrical parameters and material, loading or boundary conditions) on the responses of a structure. The first part of this article will be devoted to the presentation of the different approaches. They will be compared, in the second part, on a simple example in terms of statistical responses (average value and standard deviation of the output data) and in terms of cost of calculation. In the third part of this article, the method proposed in this work is applied to the case of calculation of a structure known as "hat-shaped", manufactured in the quasi-isotropic IM7/977-2 composite.

\section{Method for uncertainties transport}

\subsection{Monte-Carlo methods}




\section{ACCEPTED MANUSCRIPT}

The principle of the Monte-Carlo method is to choose at random (according to uniform or Gaussian distributions) values for each uncertain parameter and then to perform all the calculations associated with each set of variables. The major disadvantage of this type of approach is its cost. In fact, the number of calculations necessary to consider the extreme values (for example, the B-values of the response) could quickly become tremendous if the number $\mathrm{N}$ of variables is high (several thousands calculations if $\mathrm{N}>5$ ). Furthermore, since the results are available for a number of sets of variables in the whole range of uncertainty, it is possible to estimate directly (using traditional statistical tools) probabilistic values (average value, standard deviation ...). It should be noted that there are many alternatives to this method which are aimed at reducing the computational cost (by using an approximation of the inverse of the stiffness matrix by Neumann expansion or by using optimized random generators).

\subsection{Stochastic Finite Element Method}

Several types of methods exist under the denomination "Stochastic Finite Elements Methods" (SFEM). They are all based on the same principle: both the stiffness matrix $(K)$ and the displacement vector $(u)$ are expanded (using a perturbation method or on a polynomial base) with respect to the random variables.

For instance, using a perturbation method, the quantities under interest are expanded using Taylor series, around the mean value of the $\mathrm{N}$ uncertain variables $\alpha_{\mathrm{i}}$ (also called stochastic variables).

If a first order perturbation is considered, it is assumed that : 
$u(\alpha)=u_{0}+\sum_{i=1}^{N} u_{i} \alpha_{i} \quad u_{0}=u(0) \quad u_{i}=\left.\frac{\partial u(\alpha)}{\partial \alpha_{i}}\right|_{\alpha=0}$

The mean value and the standard variation are given by :

$$
\bar{u}=u(0) \quad \operatorname{var}(u)=\sum_{i=1}^{N} \sum_{j=1}^{N}\left(u_{i}\right)\left(u_{j}\right)^{t} C\left[\alpha_{i}, \alpha_{j}\right]=v_{1}
$$

where $C\left[\alpha_{i}, \alpha_{j}\right]$ is the covariance matrix. Note that the same expansion is used for $K$.

The second order perturbation gives :

$$
u(\alpha)=u_{O}+\sum_{i=1}^{N} u_{i} \alpha_{i}+\sum_{i=1}^{N} \sum_{j=1}^{N} u_{i j}^{I I} \alpha_{i} \alpha_{j} \quad u_{i j}^{I I}=\left.\frac{\partial^{2} u(\alpha)}{\partial \alpha_{i} \partial \alpha_{j}}\right|_{\alpha=0}
$$

The mean value and the standard variation are written as follows :

$$
\begin{aligned}
& \bar{u}=u(0)+\sum_{i=1}^{N} \sum_{j=1}^{N} u_{i j}^{I I} C\left[\alpha_{i}, \alpha_{j}\right] \\
& \operatorname{var}(u)=v_{1}+\frac{1}{4} \sum_{i, j, k, l}\left(u_{i j}^{I I}\right)\left(u_{k l}^{I I}\right)^{t}\left(C\left[\alpha_{j}, \alpha_{k}\right] C\left[\alpha_{i}, \alpha_{l}\right]+C\left[\alpha_{i}, \alpha_{k}\right] C\left[\alpha_{j}, \alpha_{l}\right]\right)
\end{aligned}
$$

The cost of this approach is due to the calculation of the derivatives by a perturbation method ( $2 \mathrm{~N}$ calculations for the first order derivative and $3 \mathrm{~N}$ for the second order derivative). A method based on an automatic derivation in the FE code is under investigation at ONERA.

The method called polynomial chaos expansion (PCN, for a $\mathrm{N}$ order polynomial expansion) [1] seems to be very effective. It is based on the expansion of $K$ and $u$ on a particular polynomial base (the base defined by the monomials is orthogonal). The base is chosen as a function of the distribution of the stochastic parameters. 
$u(\alpha)=\sum_{i=1}^{M} u_{i} \Phi_{i}(\alpha)$

with $\left\langle\Phi_{i}(\alpha) \Phi_{j}(\alpha)\right\rangle=\left\langle\Phi_{i}^{2}(\alpha)\right\rangle \delta_{i j}$ and $\langle f(\alpha) g(\alpha)\rangle=\int f(\alpha) g(\alpha) w(\alpha) d \alpha$

Using the properties of the polynomial (orthogonality), it is possible to show that :

$u_{i}=\frac{\left\langle u(\alpha) \Phi_{i}(\alpha)\right\rangle}{\left\langle\Phi_{i}(\alpha) \Phi_{i}(\alpha)\right\rangle}$

The choice of the inner product $(\langle x y\rangle)$ is a function of the distribution of the stochastic parameters. The practical implementation of this method is more complex and necessitates the computation of a number of integrals. However, the results shown in the literature seem relatively precise and obtainable for reasonable computing times.

\subsection{Surface Response based Analysis of the Variability Method}

The method proposed in the present paper could be called "experimental design numerical method" since it is strongly inspired by the experimental design methods. However, the traditional experimental design is defined to minimize the effects of the experimental errors and the noise whereas, in the present case, all the values are computation results. This method consists in building an analytical relation (most generally polynomial) between stochastic variables and the outputs using a number of runs of the complete modelling (usually a FE calculation). The choice of the values of the stochastic variables for the computations is made in order to obtain the most precise approximation. Moreover, some tools have been developed to estimate the quality of this approximation. 


\section{ACCEPTED MANUSCRIPT}

Remark : the approximation could be performed using a polynomial chaos expansion. In this case, the terms of the polynomial are calculated by eq. 7. The integrals are calculated by a Gauss integral approximation.

The proposed approach is divided into several stages (Figure 1) :

- Choice of the sets of variables to be tested,

- Realization of the calculations,

- Determination of the approximation (polynomial) function that relates the outputs to the stochastic variables,

- Calculation of the estimators of error made by the approximation,

- Calculation of the extreme values, statistical distributions of the responses, B-values... thanks to the approximation function.

One of the key points of this method is the distribution of the sets of variables to be tested. Two strategies have been developed: one based on the neural network [6] that permits to occupy as far as possible the whole hypercube defined by the range of the stochastic variables, the other one consists in occupying the tops of the hypercube as far as possible. The sets of variables being chosen, FE simulations are performed on a cluster of PC by using the natural parallelization of the problem $(3 x[\mathrm{~N}+1]$ structural analyses $)$.

The outputs are extracted from all the calculations and the relations between the uncertain variables $\alpha_{i}$ and the outputs are approximated using a polynomial form. The error evaluation of 


\section{ACCEPTED MANUSCRIPT}

the polynomial approximation is performed using various techniques of cross-validations (bootstrap [7], leave-one-out [8]). It consists in identifying $\mathrm{X}$ times the approximation using sets of calculations randomly chosen through the $\mathrm{N}$ available ones $(\mathrm{N}$ randomly or $(\mathrm{N}-\mathrm{M})$ choices among the $\mathrm{N}$ for the bootstrap and the leave-M-out methods respectively). These techniques, which allow estimating the generalization error, improve considerably the reliability of the approximation.

In practice, the approximation is first chosen as linear. If the errors are too significant (a minority of the investigated cases), it is possible to increase the degree of the polynomial interpolation. In order to avoid increasing significantly the number of monomials (384 for $\mathrm{N}=11$ with a polynomial of degree 3), the Gram-Schmidt orthogonalization algorithm is used [9]. Following this method, the monomials are iteratively ranked in order of their decreasing contribution to the output. It has been shown that in several cases it is possible to drastically reduce the errors by introducing only some additional monomials. Other approximations or regression methods are based on neural networks for highly non-linear probabilistic transfer problems.

Finally, once the approximation is identified and validated, it is very easy to obtain for each output a number of results such as extreme values (minimum and maximum of each output), the B-values (using Monte-Carlo techniques thanks to the polynomial approximation). Using the developed error approximation, it is possible to determine the confidence in these results.

\section{Comparison of the methods on a simple example}

This section is devoted to the comparison of the presented methods (in terms of average value, standard deviation and computing time). The very simple case under investigation is a $2 \mathrm{D}$ square 


\section{ACCEPTED MANUSCRIPT}

element subjected to a simple tensile test (see Figure 2). The behaviour of the material is isotropic, with a stochastic Young modulus and a deterministic Poisson's ratio $(\mu=0.31)$. The applied load $\mathrm{F}$ is equal to $100 \mathrm{~N}$ and $\mathrm{E}$ is defined by:

$$
E=\bar{E}\left(1+r_{E} \xi\right)
$$

where $\bar{E}=200 G P a$ is the mean value of the Young modulus. $\mathrm{r}_{\mathrm{E}}(=0.1)$ is the coefficient of variation. $\xi$ is a centered Gaussian random variable.

The results obtained with the different approaches are presented in Figure 2. When the number of random variables is sufficiently low $(\mathrm{N}<5)$, it is well known that for a great number of samples, the results obtained by the Monte-Carlo method (MC) could be considered as having converged (as compared with the exact solution). On this example, the $2^{\text {nd }}$ or $4^{\text {th }}$ order polynomial chaos methods (PC2 or PC4) lead to very close results as compared with the MC method. However, the cost of a calculation with the PC method is drastically less than that which the MC method. The $1^{\text {st }}$ order perturbation method is very efficient in terms of computational cost but underestimates the results.

The method proposed in the present work gives very close results as compared with the MC ones. Its computational cost is low since only some deterministic FE calculations are necessary (here 7 calculations were used to identify the approximation). The average and the variance of displacement can be calculated:

- Analytically, starting from the equations (1) and (2) for which the derivatives are very simple to calculate due to the polynomial approximation of the output

- $\quad$ By a MC method using the polynomial approximation that allows a quasi immediate estimate of the output. 
The method proposed in this paper permits to obtain very close results as compared with the other ones found in the literature, for a much lower computational cost. Moreover, the use of this method does not require any modification of the computer code since the whole analysis is performed in post-processing. Finally, it should be noted that this method permits to study material uncertainties but also geometrical ones (boundary conditions, loading ...).

\section{Influence of geometrical uncertainties}

In the following example, geometrical (dimensional uncertainties but also uncertainties on boundary conditions and loading) are investigated. The structure under investigation (called "hatshaped" structure, see Figure 3$)$ is defined by the following 9 parameters $\left(\theta_{1}, \theta_{2}, \theta_{3}, r, 1, a_{g}, a_{d}, r_{4}\right.$ and $r_{5}$ ) with uncertainty intervals of $\pm 10 \%$ for each parameter. In order to validate the proposed method, comparison with reference results obtained by a MC method (1024 FE calculations with uniform distribution on each stochastic parameter) is performed. This structure is a 8-ply quasiisotropic IM7-977-2 composite. The behaviour of the ply is described by a transverse-isotropic linear elastic model ( 5 elastic parameters). The structure is subjected to an imposed displacement. Several outputs are investigated (load, maximal stress, maximal strain, rupture criteria such as maximal admissible fiber deformation...).

Figure 4 presents all the load/displacement curves obtained thanks to the $1024 \mathrm{FE}$ calculations. The first remark is that, according to the Central Limit Theorem, even if the distribution of the stochastic variables is uniform, if the number of runs is not too small, the histogram presents a 


\section{ACCEPTED MANUSCRIPT}

Gaussian shape. Three outputs are investigated: stiffness of the structure (load/déplacement ratio), maximal strain criterion for the fibers and, finally, a damage criterion (quadratic criterion which is a function of the stress field). The first output is a global one as the other ones are evaluated by seeking, for each calculation, the maximum value on the whole structure. The statistical treatment of the MC results are presented in table 1 (average value and standard deviation of 1024 calculations as well as the minimal and maximum values).

Moreover, thanks to cross-validation approaches it is possible to evaluate the quality (confidence) of the values provided in table 1. For that, the "bootstrap" [7] method used in descriptive statistics, is well adapted. The results obtained with this method with samples of 50, 200, 512 and 1024 calculations are presented in table 2.

The method proposed in this paper has been applied and identified using a set of 20, 30 or 60 calculations. In the first part of table 3, the average values and the variations are indicated when the method is identified 100 times, starting from sets of different calculations. One can notice the good robustness of the method. It should be noted that using only one set of calculations $(0,30$ or 60 ), it is possible to obtain results equivalent to those obtained using cross-validation methods (here the method used is of type "leave-one-out"). Very similar results are obtained on the other outputs. It is also important to note that the effect of the type of distribution of the stochastic parameters on the output can be investigated using the proposed method. For instance, if a normal distribution is chosen instead of the uniform distribution, the standard deviation decreases from 14.2 to 8.6. The confidence on the values of table 3 depends obviously on the quality of the approximation (linear in this case). If the degree of confidence is low, it is possible to increase the quality of the approximation by increasing the number of monomials (chosen using the Gram- 


\section{ACCEPTED MANUSCRIPT}

Schmidt orthogonalization method, [9]. The reduction of the average quadratic error (estimated on all 1024 calculations) on the fiber rupture criterion is presented in figure 5.

\section{Conclusion}

Various methodologies may be found in the literature allowing to take into account the effect of uncertainties on the response of a structure. A method, based on the surface response has been proposed in the present paper. These various methods have been compared on simple examples in terms of response (average value and standard deviation of the output) and in terms of computational cost. The method proposed in this paper permits to evaluate a number of statistical results. This method is very simple to use since it does not need any modification of the FE code (procedure used in pre/post processing). It has been shown that the distribution of the output and the associated confidence could be evaluated using a few number of calculations $(3 \mathrm{x}[\mathrm{N}+1])$.

\section{Acknowledgments}

This work was carried out under the AMERICO project (Multiscale Analyses: Innovating Research for Composites) directed by ONERA and funded by the DGA/STTC (French Ministry of Defense) which is gratefully acknowledged. The authors would like to express their sincere gratitude to Dr. R. Valle for valuable and helpful discussions.

\section{References}

[1] Ghanem R.G., Spanos P.D., “Stochastic Finite Elements: a spectral approach”, Dover Publications, New-York, 2003 
[2] Chakraborty S., Bhattacharyya B., An efficient 3D stochastic finite element method, Int. J. Solids and Struc., 39, 2002, 2465:2475.

[3] Sudret B., Der Kiureghian A., Comparison of finite element reliability methods, Probabilistic Engineering Mechanics, 17, 2002, 337:348.

[4] Noor A.K., Starnes J.H., Peters J.M., Uncertainty analysis of stiffened composite panels, Com. Struct., 2001, 139:158.

[5] Dessombz O., Throuveriez F., Lainé J.-P., Jézéquel L., Analysis of mechanical systems using interval computations applied to finite-element methods, J. of Sounds and Vibration, 239(5), 2001, 949:968.

[6] Kohonen T., "Self Organizing Maps", Berlin, Springer, 1995

[7] Efron B., Tibshirani R., "An introduction to the bootstrap", Chapman and Hall, 1993

[8] Stone, M, "Cross-validatory choice and assessment of statistical predictions”, J. Roy. Statist. Soc. Ser. B 36, 1974, 111:147.

[9] Stoppiglia H., Dreyfus G., Dubois R., Oussar Y., "Ranking a random feature for variable and feature selection", Journal of Machine Learning Research, vol. 3, 2003, 1399:1414. 


\section{Figure captions}

Figure 1 : Principle of the proposed method

Figure 2 : Comparison between the different methods on a 2D element subjected to a uniaxial loading. (a) Presentation of the treated example, (b) Approximation used in Variability Analysis, (c) Mean value and variance (d) of the displacement.

Figure 3 : Geometry and boundary conditions of the « hat-shaped » structure

Figure 4 : Load/Displacement curve for all calculations and distribution of the load

Figure 5 : Mean square error reduction by increasing the degree of the polynomial intepolation using the Gram-Shmidt orthogonalization algorithm. 


\section{Table captions}

Table 1 : Results obtained by the Monte-Carlo method

Table 2 : Results obtained using a re-sampling method

Table 3 : Results obtained by the present method : comparison between 100 re-identification of the polynomial approximation and a re-sampling method. 


\section{Figures}

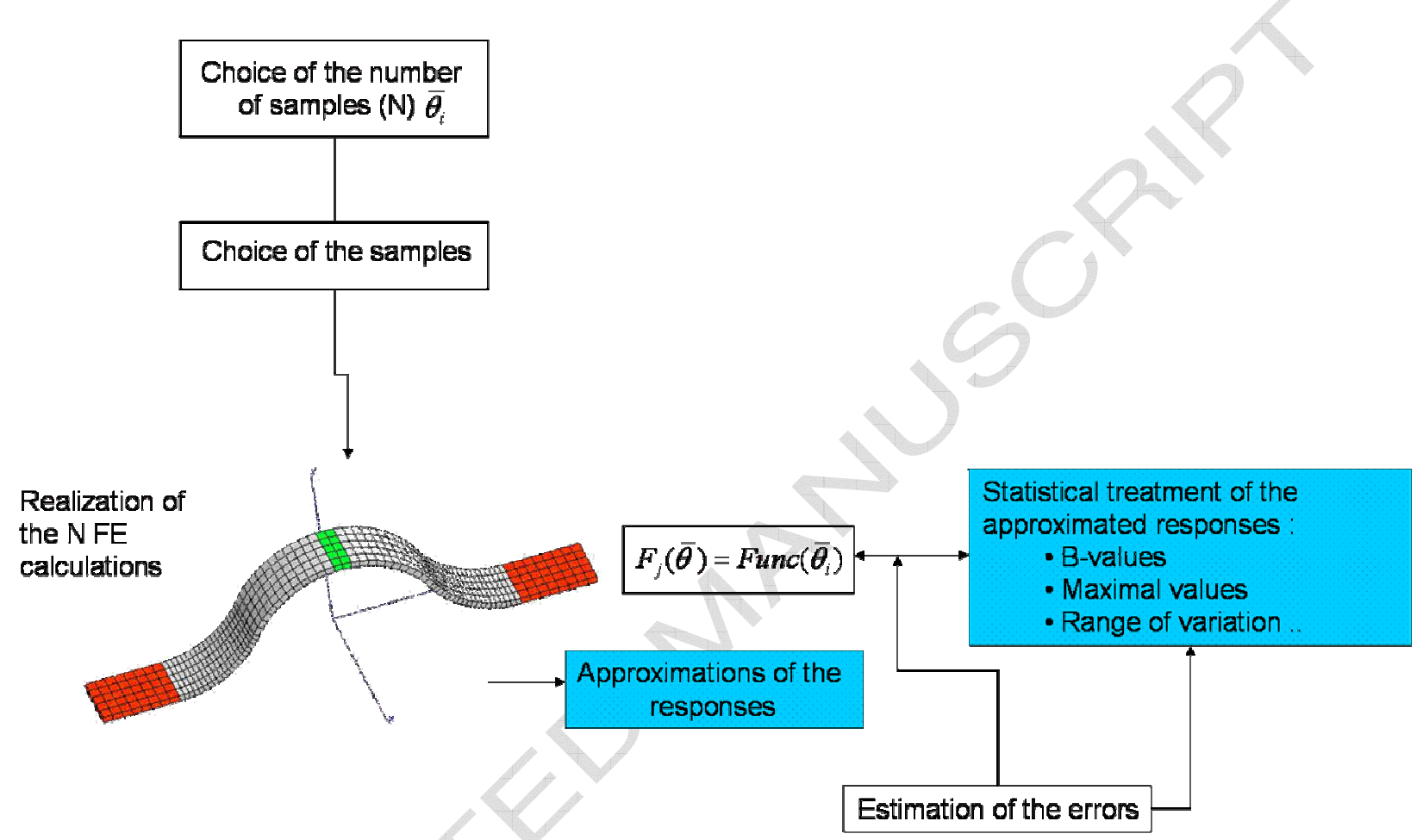

Figure 1 : Principle of the proposed method 
(a)

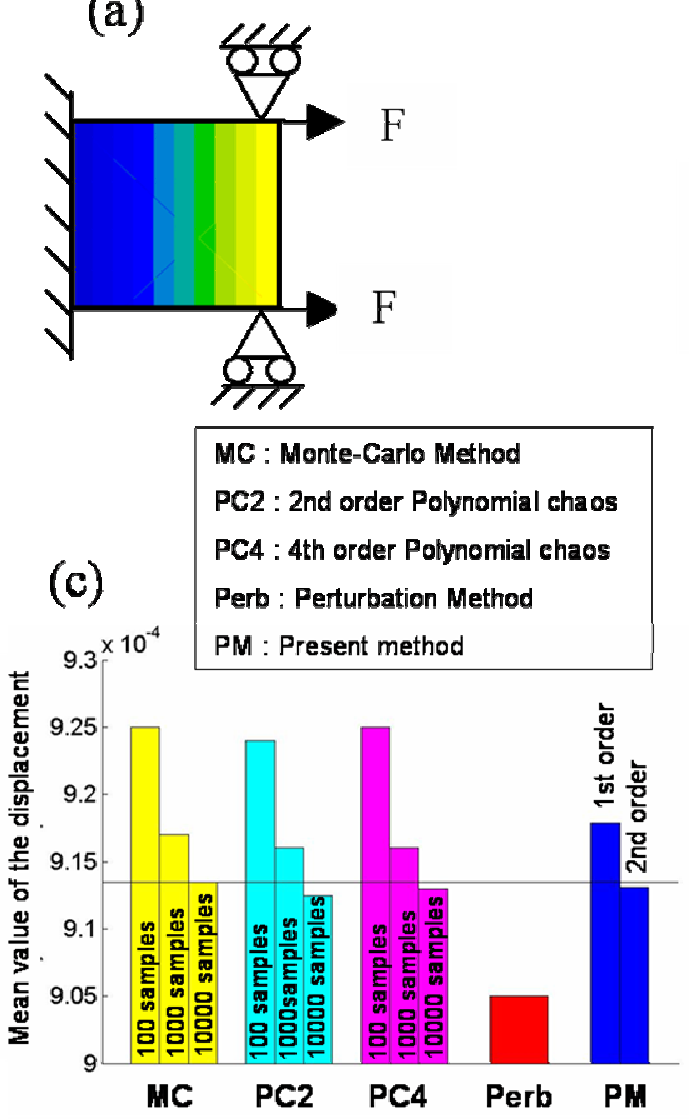

(b)

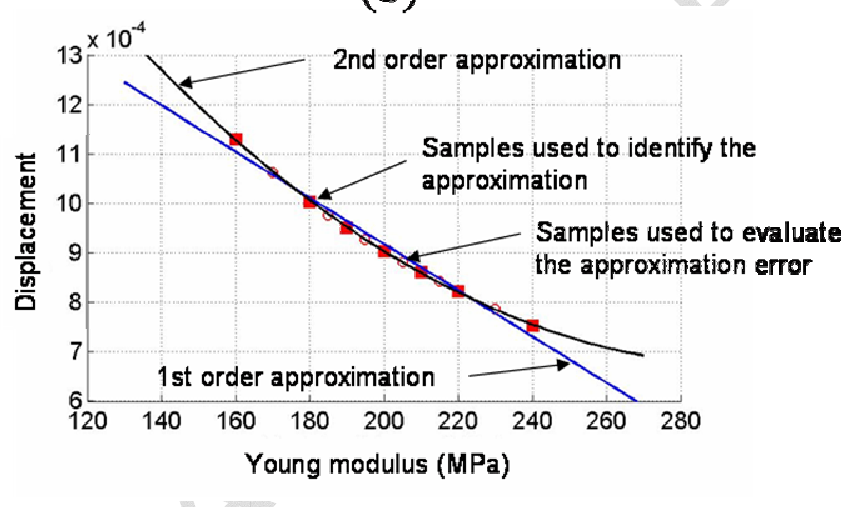

(d)

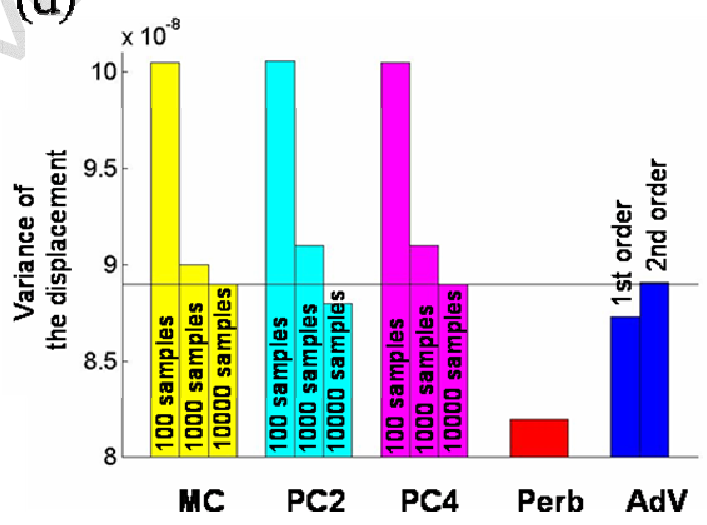

Figure 2 : Comparison between the different methods on a 2D element subjected to a uniaxial loading. (a) Presentation of the treated example, (b) Approximation used in Variability Analysis, (c) Mean value and variance (d) of the displacement. 
Boundary conditions

- simple support located at $\mathrm{a}_{\mathrm{g}}$ and $\mathrm{a}_{\mathrm{d}}$.

- pressure applied on the face defined by $\theta_{3}$.

- Nodal reactions $f_{1}$ and $f_{2}$.

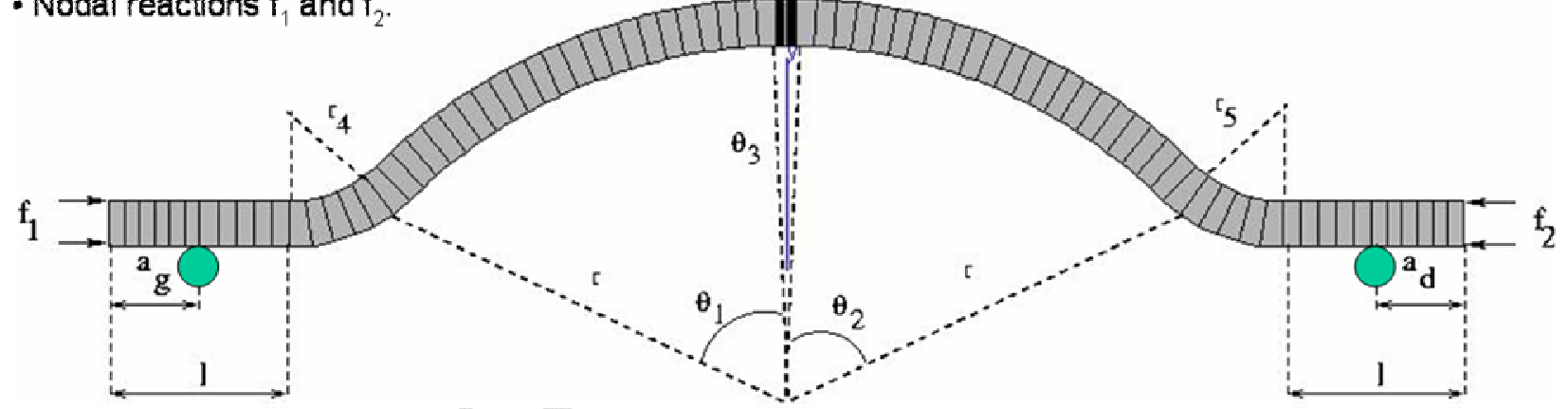

Figure 3 : Geometry and boundary conditions of the « hat-shaped » structure 


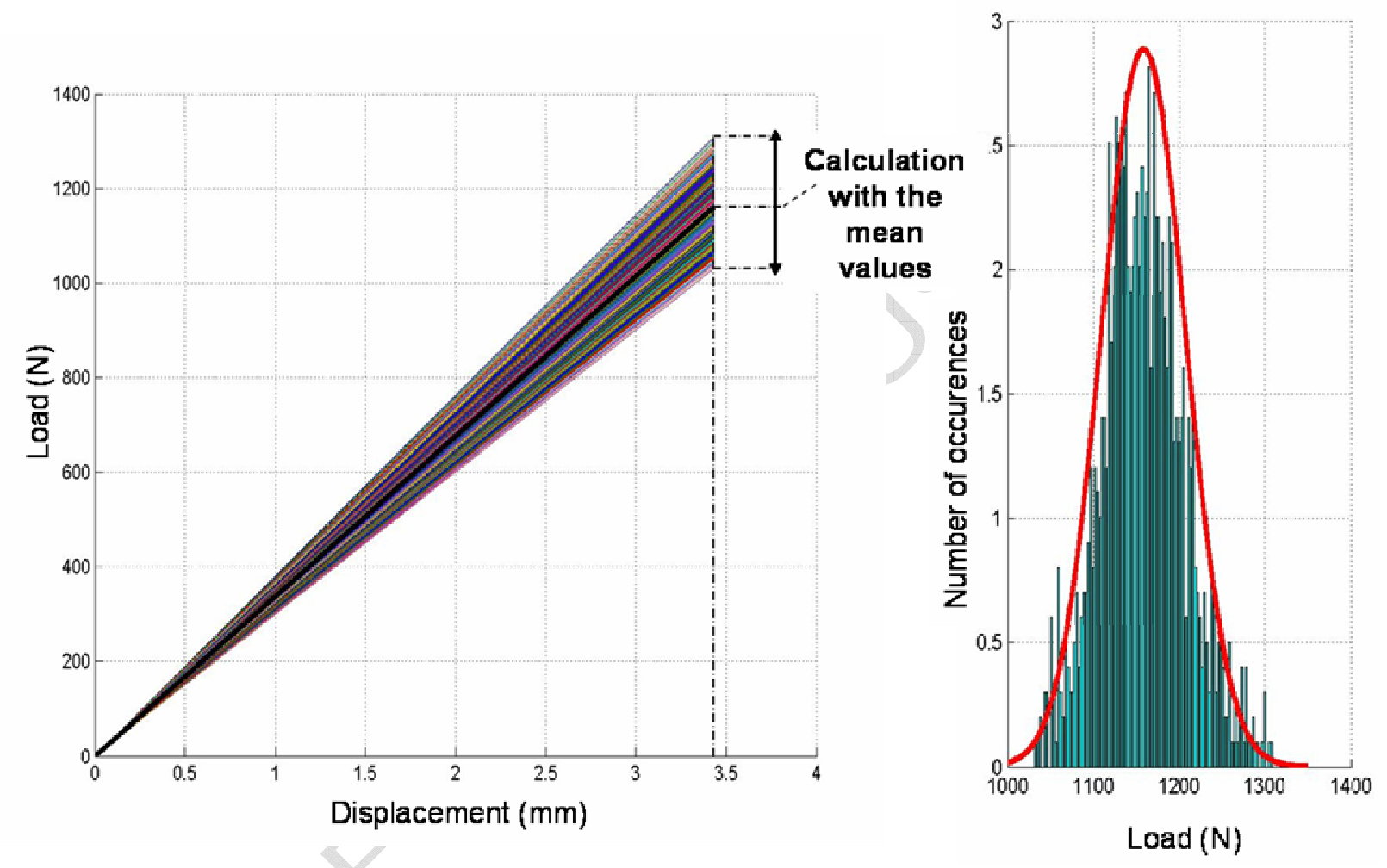

Figure 4 : Load/Displacement curve for all calculations and distribution of the load 


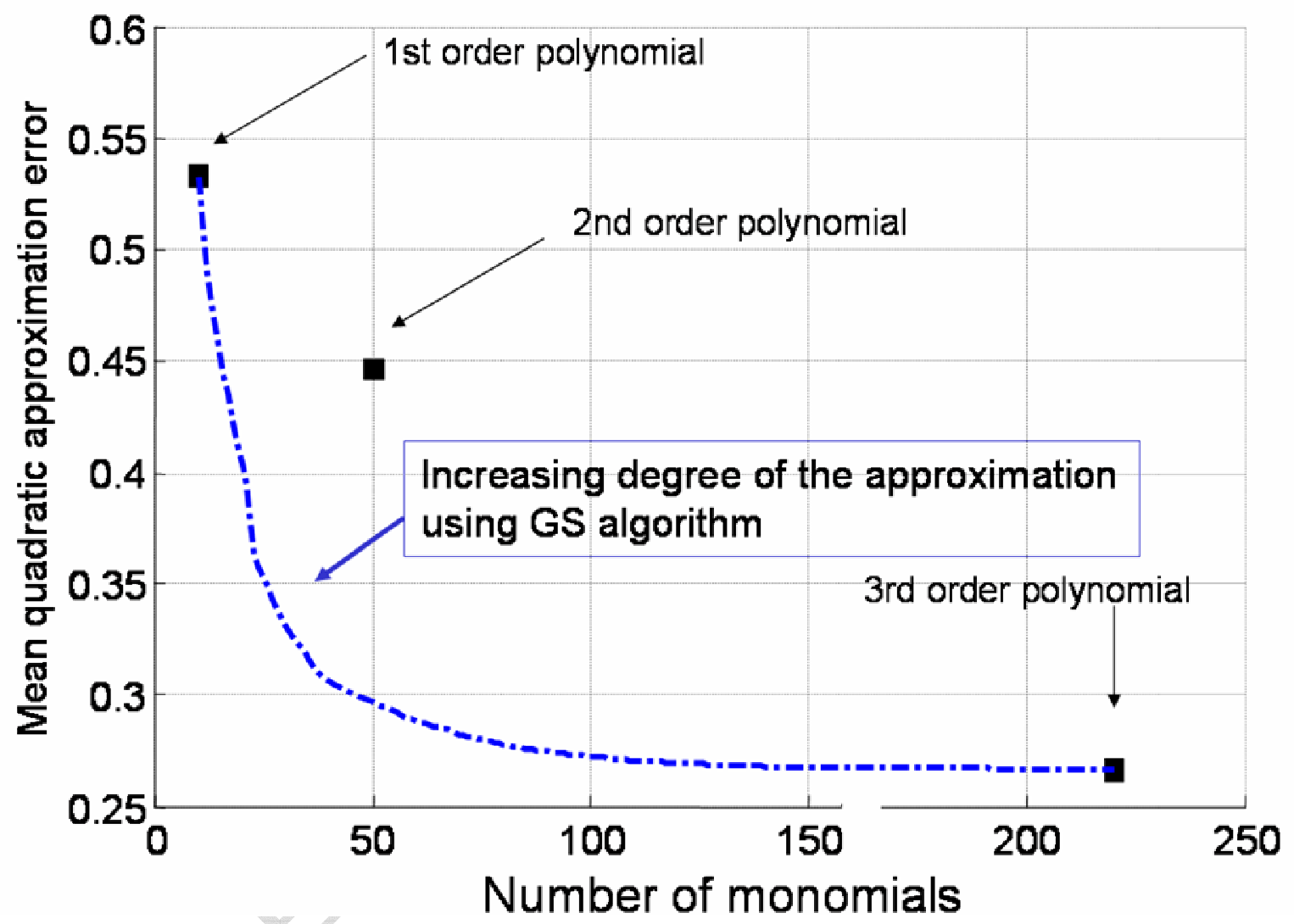

Figure 5 : Mean square error reduction by increasing the degree of the polynomial intepolation using the Gram-Shmidt orthogonalization algorithm. 


\section{Tables}

\begin{tabular}{|c|c|c|c|c|}
\cline { 2 - 4 } \multicolumn{1}{|c|}{ Monte-Carlo method } & $\begin{array}{c}\text { Mean } \\
\text { value }\end{array}$ & Standard deviation & Minimal value & Maximal value \\
\hline Stiffness (N/mm) & 335.8 & 14.2 & 298.9 & 379.5 \\
\hline Fibers strain (\%) & 1.46 & 0.04 & 1.35 & 1.58 \\
\hline Hill criterion $(\mathrm{MPa})$ & 127.5 & 3.85 & 117.1 & 139.9 \\
\hline
\end{tabular}

Table 1 : Results obtained by the Monte-Carlo method

\begin{tabular}{|c|c|c|c|c|c|}
\cline { 2 - 6 } \multicolumn{1}{c|}{ Stiffness (N/mm) } & $\mathrm{N}$ & Mean value & $\begin{array}{c}\text { Standard } \\
\text { deviation }\end{array}$ & $\begin{array}{c}\text { Minimal } \\
\text { value }\end{array}$ & Maximal value \\
\hline \multirow{3}{*}{ Bootstrap } & 50 & $336.5 \pm \mathbf{6 . 0}$ & $14.9 \pm \mathbf{6 . 2}$ & $304.7 \pm \mathbf{9 . 8}$ & $375.6 \pm \mathbf{1 5 . 8}$ \\
& 200 & $335.4 \pm \mathbf{2 . 9}$ & $14.6 \pm \mathbf{2 . 7}$ & $300.3 \pm \mathbf{5 . 7}$ & $377.7 \pm \mathbf{6 . 8}$ \\
& 512 & $335.2 \pm \mathbf{1 . 9}$ & $13.8 \pm \mathbf{1 . 8}$ & $299.7 \pm \mathbf{2 . 8}$ & $377.0 \pm \mathbf{4 . 5}$ \\
& 1024 & $335.7 \pm \mathbf{1 . 5}$ & $14.1 \pm \mathbf{1 . 1}$ & $299.5 \pm \mathbf{2 . 0}$ & $378.9 \pm \mathbf{2 . 7}$ \\
\hline
\end{tabular}

Table 2 : Results obtained using a re-sampling method

\begin{tabular}{|c|c|c|c|c|c|}
\cline { 2 - 6 } \multicolumn{1}{c|}{ Stiffness (N/mm) } & $\mathrm{N}$ & Mean value & $\begin{array}{c}\text { Standard } \\
\text { deviation }\end{array}$ & Minimal value & Maximal value \\
\hline \multirow{3}{*}{100 re-identifications } & 20 & $336.2 \pm \mathbf{0 . 8}$ & $14.5 \pm \mathbf{0 . 9}$ & $295.7 \pm \mathbf{4 . 6}$ & $376.6 \pm \mathbf{4 . 3}$ \\
& 60 & $336.2 \pm \mathbf{0 . 4}$ & $14.2 \pm \mathbf{0 . 5}$ & $298.8 \pm \mathbf{1 . 5}$ & $375.6 \pm \mathbf{2 . 3}$ \\
& 60 & $336.1 \pm \mathbf{0 . 3}$ & $14.2 \pm \mathbf{0 . 3}$ & $298.6 \pm \mathbf{1 . 0}$ & $375.6 \pm \mathbf{1 . 2}$ \\
\hline \multirow{3}{*}{ Cross-Validation } & 20 & $336.1 \pm \mathbf{1 . 8}$ & $15.4 \pm \mathbf{1 . 4}$ & $287.8 \pm \mathbf{8 . 6}$ & $384.4 \pm \mathbf{9 . 3}$ \\
& 30 & $335.9 \pm \mathbf{0 . 3}$ & $14.2 \pm \mathbf{0 . 4}$ & $297.9 \pm \mathbf{1 . 4}$ & $376.0 \pm \mathbf{2 . 5}$ \\
& 60 & $336.0 \pm \mathbf{0 . 2}$ & $14.2 \pm \mathbf{0 . 3}$ & $298.5 \pm \mathbf{0 . 8}$ & $376.5 \pm \mathbf{1 . 0}$ \\
\hline
\end{tabular}

Table 3 : Results obtained by the present method : comparison between 100 re-identification of the polynomial approximation and a re-sampling method. 